\title{
Randomized crossover sham-controlled clinical trial of targeted low-frequency transcranial magnetic stimulation comparing a figure- 8 and a round coil to treat refractory neocortical epilepsy
}

\author{
*Laura Seynaeve, *Annemie Devroye, *†Patrick Dupont, and *Wim Van Paesschen \\ Epilepsia, $* *(*): 1-10,2015$ \\ doi: 10.1111/epi.13247
}

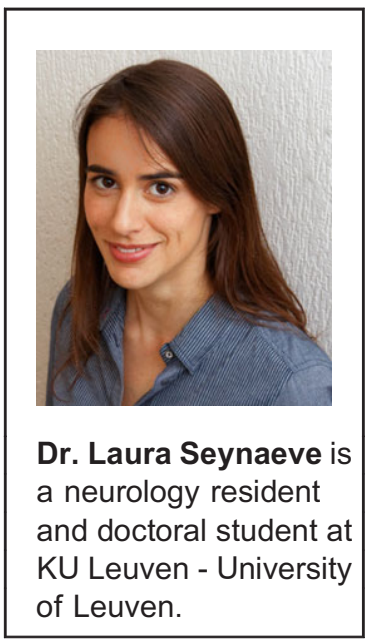

\begin{abstract}
SUMMARY
Objective: Determine the efficacy and side effects of low-frequency repetitive transcranial magnetic stimulation (rTMS) to treat refractory neocortical epilepsy and study differences in effect between a figure-8 and round coil type.

Methods: This single-center randomized sham-controlled crossover trial (NCT0 I 745952 on ClinicalTrials.gov) included I I patients with well-defined focal epilepsy. rTMS $(0.5 \mathrm{~Hz})$ was targeted to the focus during three treatment conditions consisting of 1,500 stimulations/day for 10 weekdays at $90 \%$ of resting motor threshold (rMT) followed by a 10-week observation period. Patients were randomized for the order in which the figure-8, round, and sham coil were used. Outcome assessors and patients were blinded to the type of coil used. The primary outcome measure was the percentage of seizure reduction after active rTMS treatment. Other outcome measures were responder rate, quality of life, and side effects.

Results: There was no difference between a figure-8 and round coil. None of the patients achieved an overall $\mathbf{5 0} \%$ seizure reduction. One patient responded during I month after treatment with either active coil, followed by a significant increase in seizure frequency. Another patient had a fourfold increase in seizure frequency during rTMS treatment.

Significance: This study provides evidence that rTMS is on average not effective for reducing seizure frequency. No difference in effectiveness between the different coil types was observed. It can, however, exacerbate seizures during treatment and lead to a rebound in seizure frequency after an initial reduction.

KEY WORDS: Experimental treatment, Coil type for magnetic stimulation, Seizure exacerbation.
\end{abstract}

Repetitive transcranial magnetic stimulation (rTMS) is a noninvasive brain stimulation technique that can alter the excitability of cortical regions by patterned application of a time-varying electromagnetic field. ${ }^{1}$ Inhibitory protocols

Accepted October 9, 2015.

*Department of Neurology, Laboratory for Epilepsy Research, University Hospitals \& KU Leuven, Leuven, Belgium; and †Laboratory for Cognitive Neurology, KU Leuven, Leuven, Belgium

Address correspondence to Laura Seynaeve, Department of Neurology, University Hospitals Leuven, Herestraat 49, 3000 Leuven, Belgium. Email: laura.seynaeve@uzleuven.be

Wiley Periodicals, Inc.

(C) 2015 International League Against Epilepsy such as low-frequency rTMS seem to hold promise for epilepsy treatment. Until now, 13 studies with a total of 196 patients undergoing active treatment showed mixed results. $^{2-14}$ This could be due in part to the variability in inclusion criteria and treatment protocols. Based on individual trials and the meta-analysis of Hsu et al., ${ }^{15}$ it was demonstrated that low-frequency rTMS is especially promising for patients with cortical dysplasia or neocortical epilepsy, if the stimulation was targeted to the epileptic focus. rTMS aimed at the vertex was not effective, ${ }^{11,15}$ probably because the epileptogenic zone was not targeted. rTMS of mesial temporal structures was not effective ${ }^{15}$ 


KEY POINTS
- Low frequency rTMS is overall ineffective to reduce
seizure frequency in a 2-month period following active
treatment
- No difference was seen between treatments using a
figure-8 or a round coil positioned over the focus
- rTMS can cause rebound seizures after an initial
response
- rTMS can acutely exacerbate seizures

because these structures are too deep to be affected directly by rTMS.

In most studies, the target of stimulation was the most active point in the 10-20 or 10-10 electroencephalography (EEG) system. However, in one patient, ${ }^{12}$ the ictal EEG and single-photon emission computerized tomography coregistered to MRI (SISCOM) data were used to define the focus, and neuronavigation was used to position the TMS coil. This patient experienced a $90 \%$ seizure reduction. This was the only study ${ }^{12}$ to date using neuronavigation. Treatment protocols also differed in their stimulation frequencies and intensities, but those differences were not clearly associated with outcome. ${ }^{15} \mathrm{~A}$ wide range of coil types, both commercially available and custom-made, have been used. The number of rTMS pulses a day, days of treatment, and spread of treatment sessions over time were also diverse, ranging from $100^{3}$ to $3,000^{12}$ stimuli per day with treatments administered daily in most studies but biweekly in others. ${ }^{3,6}$ Studies using higher numbers of stimuli obtained better results than those using lower numbers. To capture the full potential of rTMS to reduce seizure frequency, we decided to incorporate all factors that have been shown to be beneficial, namely low-frequency stimulation targeting the epileptic focus using neuronavigation, inclusion of patients with well-delineated neocortical epilepsy, and a high number of total stimuli during a treatment block.

A factor that has not been studied to date is whether the effect of rTMS is influenced by the coil type used. All studies with stimulation over the epileptic focus used a figure-8 coil. ${ }^{4,6,8,10,12-14}$ With use of the figure- 8 coil, the maximal stimulation will occur near its center, whereas with the round coil, inhibition of brain tissue surrounding the center of the coil is expected. Both could be effective, since epilepsy is not only a problem of hyperexcitability of the focus but also of failure to prevent spread to neighboring brain regions. It has been shown that the round coil-when positioned over the focus-is more effective than the figure- 8 coil for aborting epileptic discharges in patients with frontal lobe epilepsy. ${ }^{16}$ No study to date, however, has evaluated the potential to reduce seizures when targeting the epileptic focus with a round coil. To investigate whether there are differences in effect between the figure- 8 and the round coil in individual patients, we performed a double-blind randomized sham-controlled crossover clinical trial. The primary aim of our study was to validate rTMS as a clinical tool to treat selected patients with refractory neocortical epilepsy.

\section{MeTHODS}

\section{Participants}

Eligible participants were 16-75 years old, with refractory focal epilepsy and a single epileptogenic zone, which was determined during a presurgical investigation. Resection was not an option due to the proximity of eloquent cortex or the patient declined surgery. The seizure frequency was at least four per month and was recorded reliably in a seizure diary by the patient or a caregiver. Antiepileptic drugs (AEDs) were kept unchanged throughout the study. Exclusion criteria were the exclusion criteria for TMS (intracranial metal devices, pacemakers, ICDs, and so on), nonepileptic seizures, rapidly progressive medical diseases, suicidal ideation, pregnancy, and alcohol or drug abuse. Patients were referred by epileptologists involved in multidisciplinary presurgical evaluation. No information about the details of the randomization protocol was given to referring physicians. Previous failed epilepsy surgery was not an exclusion criterion.

\section{Study design}

The trial utilized a prospective, randomized, double-blind crossover design using three different coils to compare a figure- 8 and round coil versus sham in each patient. The order in which the three coils were used in each patient was randomized using a computerized random number generator and a permutation for each block of three patients. After 8 weeks of baseline evaluation on a stable drug dose, patients underwent 2 weeks of stimulation (10 sessions) with 1,500 stimuli a day at a frequency of $0.5 \mathrm{~Hz}$, followed by a 10-week observation period. Treatments were performed in an outpatient setting, and sessions lasted about an hour each day. This treatment was repeated twice, using the figure- 8 coil, the round coil, or the sham coil during the treatment sessions. Patients were screened for inclusion by one epileptologist (WVP). The treatment was always administered by the same investigator (LS) who was blinded to seizure count. Seizures diaries were checked by the study nurse (AD) every 5 weeks and assessed by the epileptologist (WVP) at the end of each treatment period. Both assessors were blinded to the order of the treatments. The study nurse also systematically inquired about side effects after each session. ${ }^{17}$ During baseline evaluation and at the end of each observation period Quality Of Life In Epilepsy-31 (QOLIE-31), ${ }^{18}$ the Columbia Suicide Severity Rating Scale (CSSRS), and global impression of change-scales were rated. In order to assess if patients could be blinded in a 


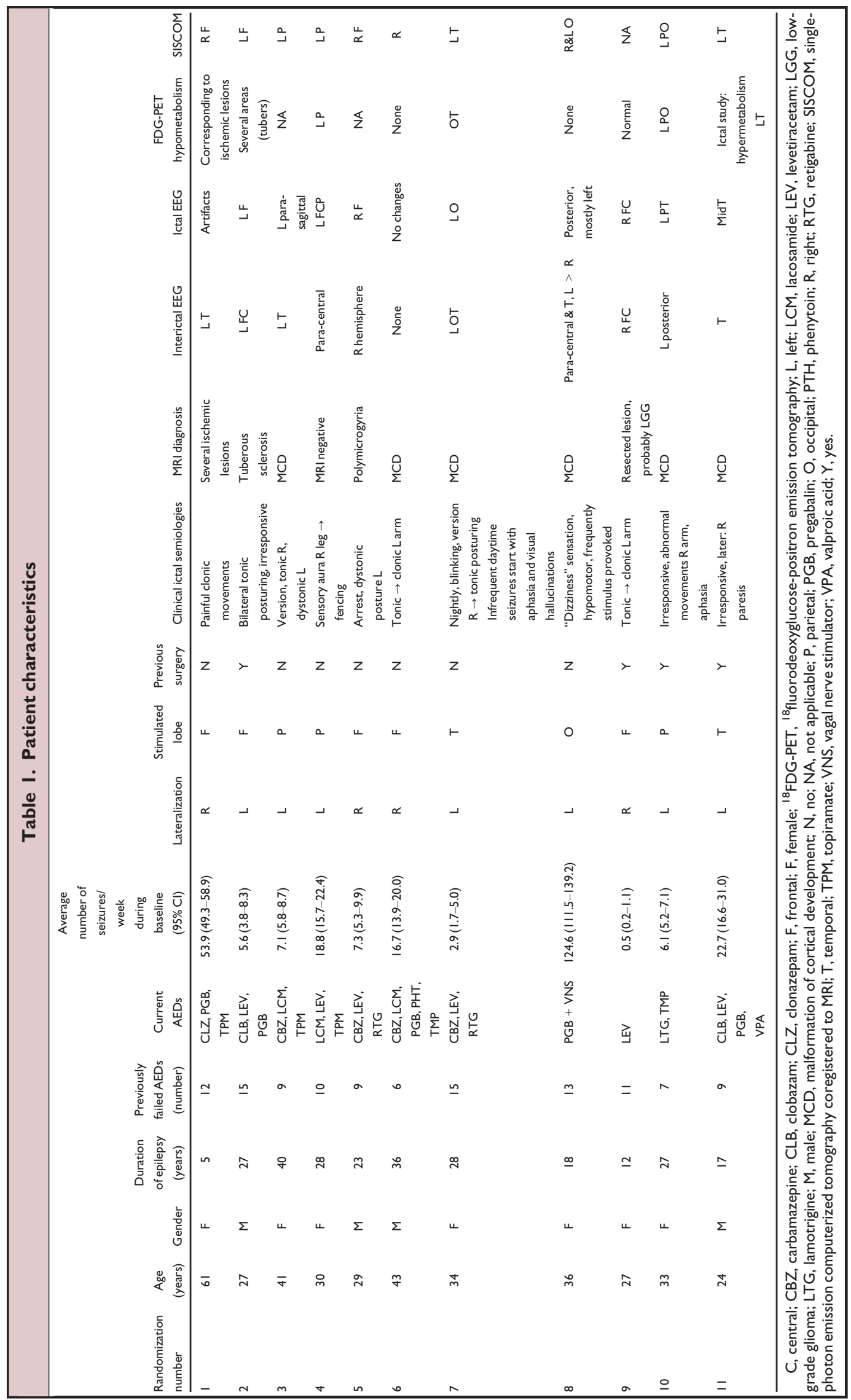




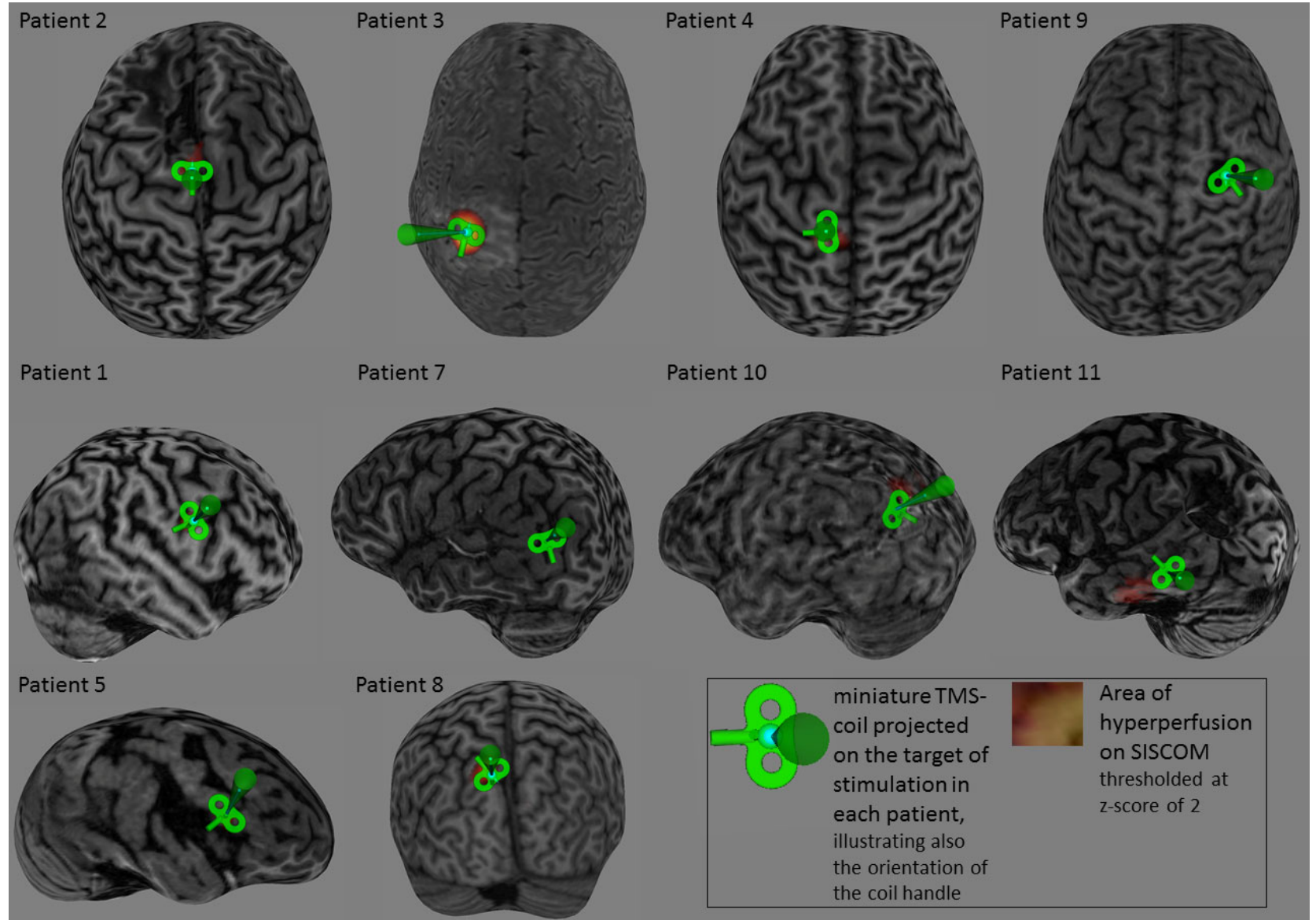

Figure I.

Target of stimulation in individual patients, based on all available data of a presurgical workup. A miniature TMS coil is positioned over the target of stimulation on a $3 \mathrm{D}$ rendering of the brain. The orientation of the coil during rTMS is illustrated. SISCOM hyperperfusion is visualized in an orange color.

Epilepsia (c) ILAE

satisfactory manner in a crossover trial, they were asked to write down after the first day of each treatment block if they thought a real or sham coil was used that day. These data remained in a sealed envelope until all statistical analyses were performed. The center of the ictal onset zone, as determined by the multidisciplinary epilepsy surgery team based on magnetic resonance imaging (MRI), video-EEG, fluorodeoxyglucose-positron emission tomography (FDGPET), and SISCOM data (Table 1, Fig. 1) was chosen as the target to aim the center of the TMS coil. Neuronavigation using BrainSight (Rogue Research, Montreal, QC, Canada) was used for coil placement, and continued feedback was used during the whole stimulation session. A Magstim Rapid2 (Magstim, Whitland, United Kingdom) was used, with standard 70-mm figure-8 coil, a standard round 90-mm coil, and the commercially available sham coil. Intensity was set as $90 \%$ of the resting motor threshold (rMT), as determined at the onset of the study using the figure- 8 coil. rMT was measured by first localizing the motor hotspot by mapping a $5 \times 5 \mathrm{~cm}$ area around the hand knob, using neuronavigation, with electromyography (EMG) recordings from the abductor pollicis brevis muscle (APB). Intensity was first increased until motor evoked potentials (MEPs) could be provoked to determine the hotspot. Next, intensity was lowered in steps of $1 \%$ to determine the lowest intensity needed to provoke 5/10 MEPs on EMG. We preferred this protocol to using a different intensity when using the round coil or adapting the threshold each day, to minimize chances of inadvertent unblinding of the patients. The orientation of the coil was chosen so that it was perpendicular to the nearest important sulcus, as determined by a threedimensional (3D) reconstruction of the patients anatomic brain MRI (Fig. 1). The choice between clockwise and counterclockwise current flow with the round coil depended on the hemisphere that was targeted and was based on the optimal orientation for eliciting motor responses. ${ }^{19}$ The 1,500 stimuli a day were divided in three blocks, with short breaks in between to allow for coil cooling and to check if the neuronavigation system was still correctly calibrated. All patients used foam earplugs (3M EAR Classic). The trial 
was approved by the ethical committee of the University Hospitals Leuven and registered as NCT01745952 on ClinicalTrials.gov. Written informed consent was obtained from all patients.

\section{Statistical analysis methods}

Based on a coefficient of variation equal to 1 derived from data reported by Fregni et al., ${ }^{10} 29$ patients were needed to have at least $80 \%$ power to show a reduction of $50 \%$ between the active coil and the sham conditions based on a two-sided $t$-test for lognormal data (since two comparisons are performed, alpha is set at 0.025 ). For the main comparison of both coil conditions versus sham, 18 patients were needed. These calculations were performed under the worst case scenario of no correlation between the conditions (i.e., no patient effect). Because it is reasonable to expect a patient effect on the seizure rate, we aimed at including at least 20 patients in the study. Recruitment was slower than anticipated and, therefore, it was decided to terminate the study before the planned number of subjects was included. Note that the number of daily measurements per patient per condition was large enough to guarantee at least $80 \%$ power for the within-patient comparisons. First, for each patient separately the number of seizures has been compared between conditions with a Quasi-Poisson regression model for count data followed by pairwise comparisons with Tukey adjustments for multiple testing. Data were also analyzed using negative binomial regression model as a sensitivity analysis, since this model gives more weight to lower counts. Second, the aggregated data over all analyzed patients were compared between conditions using a negative binomial model with extra correction for overdispersion by adding a multiplicative overdispersion parameter using Pearson chi-square statistics. The analysis on the aggregated data did not take into account the withinpatient correlation between the different treatment conditions. The analyses on patient level as well as on all patients combined did not model the evolution over time within each condition or the order of the treatment conditions within a patient. An analysis of carryover effect was planned but could not be performed due to small numbers. A corrected p-value of 0.05 was considered significant. Analyses have been performed using SAS software (version 9.2, Windows).

\section{RESULTS}

\section{Study population}

Fifteen patients were screened for inclusion, of which 11 agreed to participate (patients 1-11) (Fig. 2). Demographics and clinical data of the randomized patients are given in Table 1. Patients included in our study had refractory epilepsy with a median of 24 seizures/month (range: 18/day to 2/month: one patient had lower seizure frequency than specified in inclusion criteria). They had failed on average 11

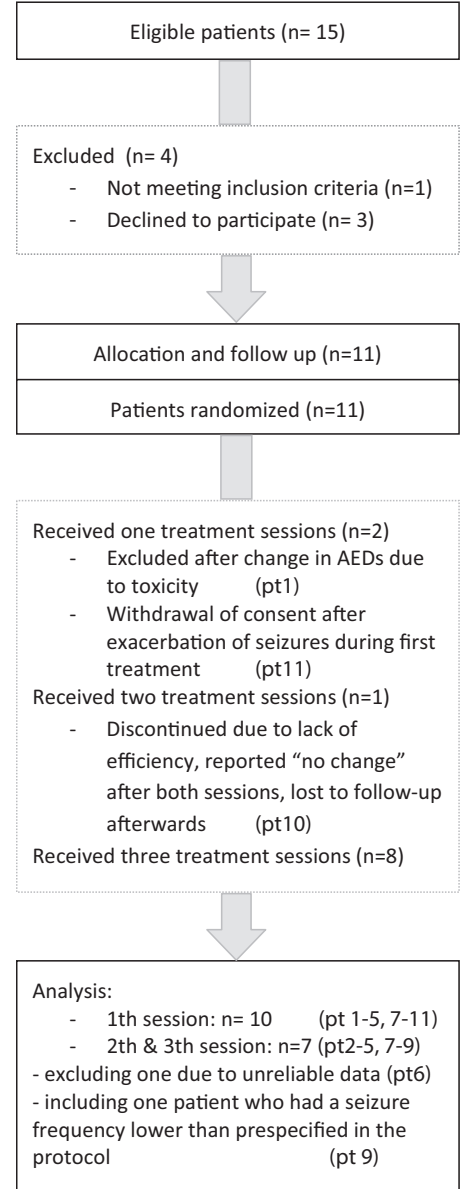

Figure 2.

CONSORT flowchart of recruitment and selection. AED, antiepileptic drug; pt, patient. Patients were numbered in order of randomization.

Epilepsia (C) ILAE

$( \pm 3)$ AEDs and were taking three AEDs (range 1-5) during the study. Four patients had undergone unsuccessful epilepsy surgery, with incomplete resections near eloquent cortex. Randomized patients were included from November 2012 until January 2014. The 11 randomized patients underwent at least one full session of rTMS with the allocated coil and kept a seizure diary during each 12 -week treatment period. The data of one patient (patient 6) were not considered for further analysis since the seizure diary did not include the number of seizures per day on several occasions. Three patients did not finish the whole protocol. Patient 1 was excluded after the first session since the AED regimen was changed due to toxicity. Patient 10 discontinued the study after two treatment sessions, since she experienced the sessions as painful and not effective. Data of the observation period after the second (sham) treatment block were not available. Patient 11 discontinued because of a fourfold increase in seizure frequency during the second week of first treatment session with a figure-8 coil (Fig. 3). 


\section{Efficacy}

No difference in mean seizure rate could be detected in any of the conditions compared to baseline or between any of the conditions. After corrections for differences in baseline seizure count, results remained unchanged. Using a negative binomial regression model resulted in the same findings. To rule out an effect of shorter duration, a post hoc analysis restricted to the first month of each condition was performed. Again no change in average seizure frequency was detected. Statistically significant changes in the seizure frequency in individual patients between different treatment conditions were seen in patients 4 and 8 (Table 2 and Fig. 3). Patient 4 had a $18 \%$ seizure reduction after treatment with a figure- 8 coil, $48 \%$ seizure reduction after round coil treatment, and a $44 \%$ reduction in the subsequent treatment condition using the sham coil. In patient 8 , worsening in overall seizure frequency during the study period was significant, despite a clinically meaningful weekly seizure frequency reduction of $>50 \%$ during the first month after each active treatment. After this initial seizure frequency reduction, seizure frequency increased above the $95 \%$ confidence interval (CI) of baseline seizure frequency during 18 weeks following the reduction after the treatment with a round coil (including the following period of sham treatment), and for $>20$ weeks after treatment with the figure- 8 coil (data not shown in Fig. 3).

\section{Secondary outcome measures}

Seven patients were able to fill out questionnaires. The baseline average quality of life based on the QOLIE-31 was $50 / 100$ (with higher scores meaning better quality of life). In three of six patients there was an improvement in QoL with medium effect size ${ }^{20}$ after using the round coil, three of five reported improvement after treatment with the figure- 8 coil, and one of five after sham treatment. The latter patient reported an improvement compared to baseline after each treatment, with the smallest effect size after sham treatment. One patient had worse scores after treatment with the round and the sham coil. No relation with the treated hemisphere was seen. No suicidal ideations were reported during the study. When rating the global impression of change, five of six patients reported no change after sham-treatment, four of seven after figure- 8 treatment, and five of eight after treatment with the round coil. A moderate unfavorable evolution was reported by three different patients after one treatment session-sham, figure-8, and round coil treatment, respectively. Patient 8 reported first a very favorable response followed by an unfavorable one, as can be seen also in the seizure evolution over time (Fig. 3, Table 2). We checked allocation concealment by asking the patients to guess the coil used in each session. For the first treatment session, correct guessing of the allocated treatment was not higher than could be expected by chance (Binomial test, $p=0.26$ ); for subsequent coils, patients could guess the allocated treatment better than could be expected by chance.

\section{Adverse events}

The most important adverse effect noted in this study was a negative effect on seizure frequency. In patient 8 , there was a clear increase in seizure frequency after an initial reduction, and this increase was maintained up to 20 weeks after the end of the study. This rebound in seizure frequency was not a gradual process but rather an abrupt change from one day to the next, and it was accompanied by severe headache during 1 week in this patient with occipital epilepsy. Patient 11 had a marked increase in seizure frequency during the days of rTMS. Electroclinically these seizures were comparable to the habitual seizures of the patient. The seizures were more frequent during the actual stimulation and in the hours following treatment. One patient experienced hearing problems after stimulation, which was helped by placing some pads between the ear and the coil. Four patients experienced headache. Two patients experienced fatigue with active treatment; two other patients experienced fatigue with sham treatment. One patient reported difficulties concentrating. Side effects were minor according to the patients, except in one patient in whom the headache started within minutes of active treatment and felt like "the operative scar was going to explode."

\section{Discussion}

We report the data of a double-blind sham-controlled crossover trial of low frequency rTMS in patients with refractory neocortical epilepsy. We found no difference between targeted rTMS over the focus using a figure- 8 or a round coil.

Surprisingly, our study demonstrated no overall effect on seizure frequency using $0.5 \mathrm{~Hz}$ rTMS. This negative finding for targeted rTMS using a figure- 8 coil is in contrast with the effect size of 0.71 (with a $95 \%$ CI at $0.30-1.12$ ) in the meta-analysis of Hsu et al. ${ }^{15}$ and of 0.64 in the study of Sun et al. ${ }^{14}$ Our protocol was designed to incorporate factors known to be associated with a positive outcome: a 2 -week $0.5 \mathrm{~Hz}$ paradigm at an intensity $90 \%$ of rMT like the latter study combined with detailed delineation of the focus including ictal single-photon emission computed tomography (SPECT) ${ }^{12}$ and neuronavigation to position the coil.

Our protocol was most similar to the one described in the study of Sun et al., ${ }^{14}$ which is the largest study reporting positive results to date. It is not described in their study how many patients experienced a $50 \%$ seizure reduction, but the number is probably high given that 11 of 31 patients in the active treatment group were seizure-free at the end of the 8 -week observation period. They used a custom-made figure- 8 coil with $87 \mathrm{~mm}$ loops, whereas we used the Magstim figure- 8 coil with $70 \mathrm{~mm}$ loops. In their study, patients took on average two AEDs, which is lower than three AEDs taken in our patients' sample. This could explain variable responses to rTMS. ${ }^{21,22}$ The effect of a combination of 


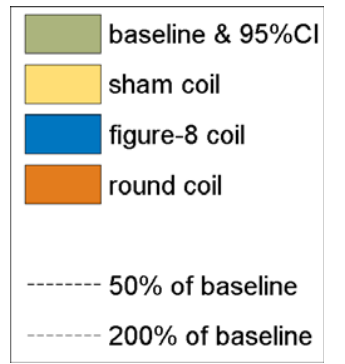
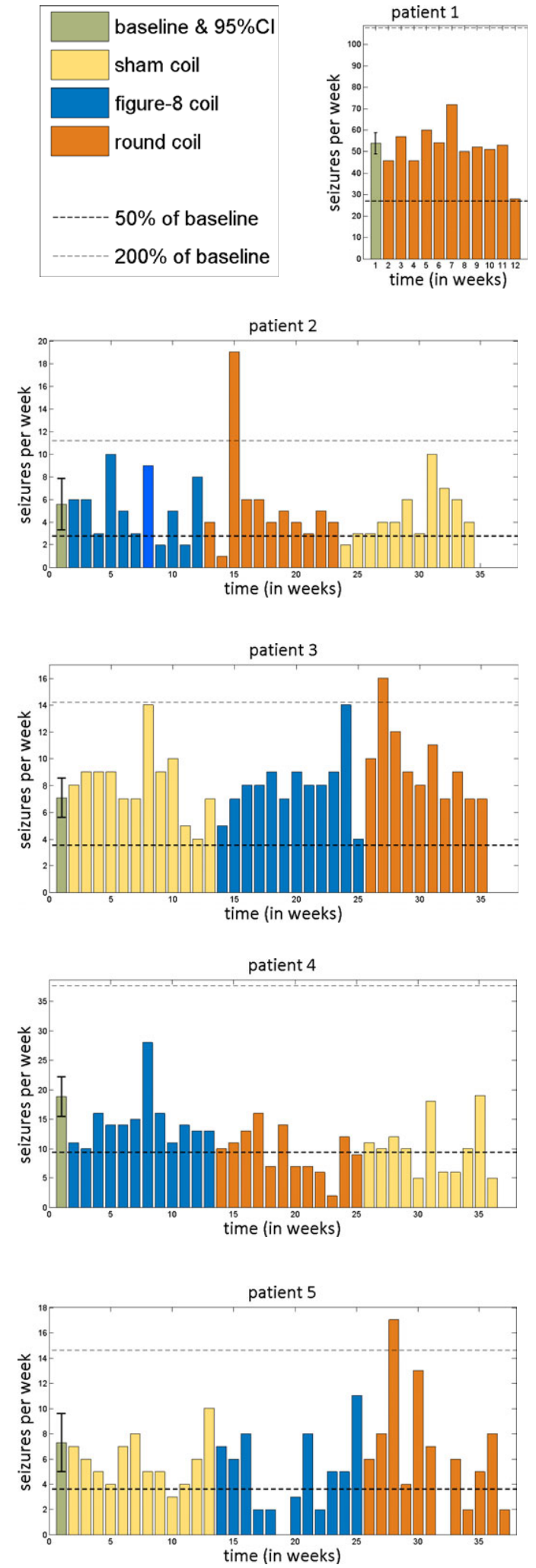

Figure 3.

Evolution of weekly seizure frequency over time in individual patients. Epilepsia (C) ILAE
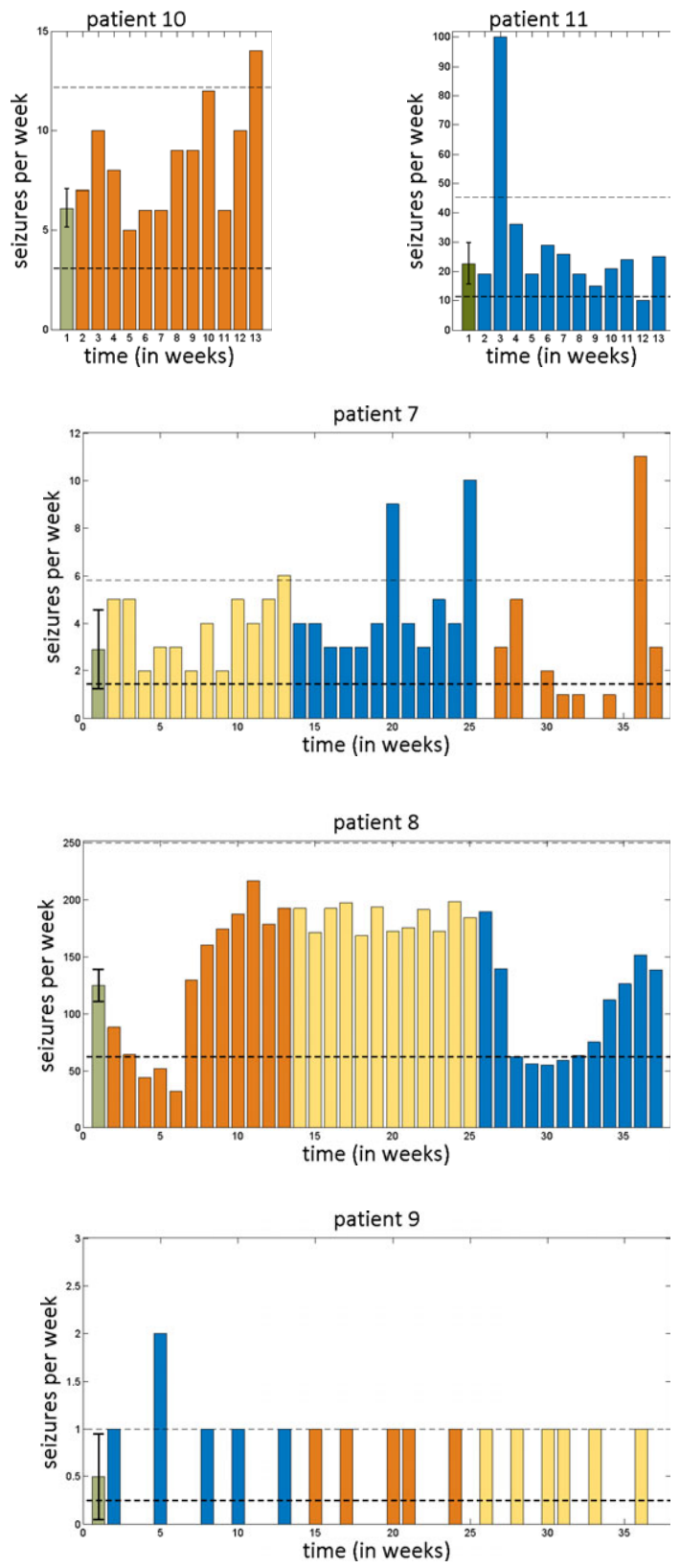


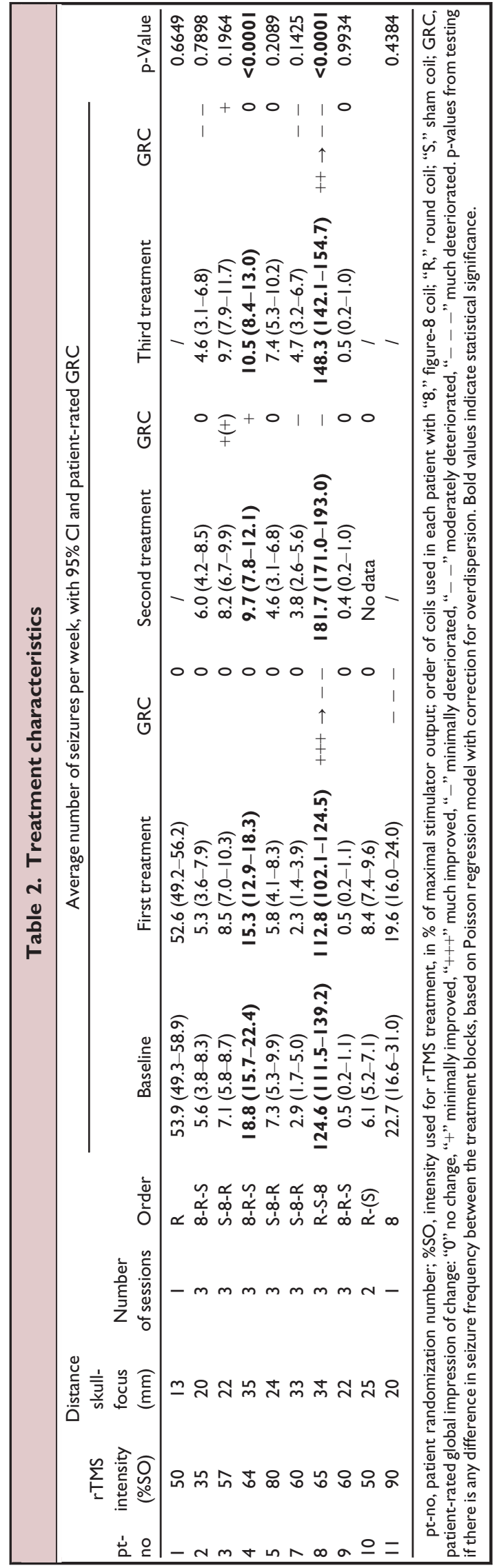

AEDs on the ability of rTMS to induce brain plasticity has not been studied, but we speculate that high doses of combined AEDs may limit the effect of rTMS to induce synaptic alterations. Of note, the patient in our study who experienced a transient improvement after both active coils took only one AED.

An important determining factor for rTMS response is the etiology of the epilepsy. Several other trials using rTMS have been negative, ${ }^{2,4,6,7,9,11,12}$ but those trials often used nonfocal stimulation ${ }^{2,7,9,11,12}$ and included patients with both focal and multifocal epilepsy $6,9,11,12$ or neocortical and mesial temporal epilepsy. ${ }^{2,4,7,9,11,12}$ Patients with mesial temporal lobe epilepsy or multifocal epilepsy were, therefore, excluded from our study. Encephalomalacia was present in 35\% of the population of Sun et al., ${ }^{14}$ but not in our population. Fregni et al. ${ }^{8,10}$ reported positive results in two trials in patients with polymicrogyria or nodular heterotopia. We included several patients with malformations of cortical development, but not these subtypes. In addition, procedural details can affect how the brain is stimulated in otherwise identical protocols. One example is the way the motor threshold is defined. Using neuronavigation and EMG results in less chance to overestimate the rMT and thus can lead to a lower intensity used for stimulation compared to previous studies that relied on anatomic surface markers.

Variability in rTMS response has also been ascribed to age, gender, time of day, previous physical and mental activity, genetic factors, ${ }^{23}$ brain states during stimulation, ${ }^{24,25}$ short breaks in sessions, ${ }^{26}$ and corticospinal excitability. ${ }^{27}$ Moreover, the effect on individual neurons depends on their orientation relative to the induced electrical field, and in epileptogenic lesions the architecture of the neuronal elements can be different from that of other brain regions. Part of the difference in response to rTMS between our study and the study of Sun et al. ${ }^{14}$ might reflect reported inherent neurophysiologic differences between Chinese and Caucasians. ${ }^{28}$ This means that identical and seemingly identical rTMS protocols can have varying effects on an individual's brain.

Even for well-established stimulation protocols over the motor cortex, large variability is seen, with only $25-36 \%$ of healthy subjects showing the expected changes in MEP amplitudes in both directions. ${ }^{27,29}$

The variability in response in different studies using rTMS can be explained at least partially by the small sample sizes and variability in rTMS protocols in combination with a large interindividual variability in response to TMSinduced plasticity.

In our study, one patient experienced a $>50 \%$ reduction in seizure frequency during the month following each active treatment, but afterwards a rebound phenomenon was observed with a clear increase in seizure frequency. This increased seizure frequency compared to baseline, was maintained up to 20 weeks after the end of the study. This is 
to our knowledge the first report of rebound seizures after successful treatment with rTMS. Moreover, our low-frequency protocol exacerbated seizure frequency acutely in one individual.

rTMS has been considered as a treatment that is well-tolerated when respecting the guidelines. ${ }^{30} \mathrm{We}$ agree that this is true in the majority of cases, but in patients with preexisting allodynia over a scar from previously failed epilepsy surgery, rTMS may be very painful. Half of our patients had some improvement in QoL scores, consistent with the observation that rTMS can influence psychological functioning irrespective of seizure reduction. ${ }^{14}$ Improvement in QoL was not dependent on the stimulated hemisphere. It has to be noted that the QOLIE-31 questionnaire specifically asked about the 4 weeks before administration of the questionnaire and we thus recorded changes occurring 812 weeks after the first stimulation session. Our patient 8 had a significant improvement in QoL 1 month after treatment, but not at the time when we administered the QoL questionnaire.

\section{Conclusion}

We report the first pilot study of neuronavigated rTMS in the treatment of focal neocortical epilepsy comparing a figure- 8 and a round coil, but found no difference in efficacy. To our surprise, we found no clear overall effect on seizure frequency. We did see one acute exacerbation during active treatment and one patient who responded during 1 month, with deterioration afterward. Because our study was small - as most rTMS studies in epilepsy - a large multicenter trial will be needed to determine the position of neuronavigated rTMS in the treatment of refractory focal neocortical epilepsy.

\section{ACKNOWLEDGMents}

This work has been supported by a grant from the Institute of Innovation by Science and Technology Flanders (IWT), project number 090850. Our gratitude goes to Dr. R. Hauman, Dr. B. Legros, and Dr. S. Ferrao Santos, who referred patients to participate in the study, the New York State Psychiatric Institute for the use of the CSSRS, and the Epilepsy Therapy Group for the use of the QOLIE-31.

\section{Disclosure}

None of the authors has any conflict of interest to disclose. We confirm that we have read the Journal's position on issues involved in ethical publication and affirm that this report is consistent with those guidelines.

\section{REFERENCES}

1. Ridding MC, Rothwell JC. Is there a future for therapeutic use of transcranial magnetic stimulation? Nat Rev Neurosci 2007;8:559-567.

2. Tergau F, Naumann U, Paulus W, et al. Low-frequency repetitive transcranial magnetic stimulation improves intractable epilepsy. Lancet 1999;353:2209.
3. Menkes DL, Gruenthal M. Slow-frequency repetitive transcranial magnetic stimulation in a patient with focal cortical dysplasia. Epilepsia 2000;41:240-242.

4. Theodore WH, Hunter K, Chen R, et al. Transcranial magnetic stimulation for the treatment of seizures: a controlled study. Neurology 2002;59:560-562.

5. Tergau F, Neumann D, Rosenow F, et al. Can epilepsies be improved by repetitive transcranial magnetic stimulation?-interim analysis of a controlled study. Suppl Clin Neurophysiol 2003;56:400-405.

6. Daniele O, Brighina F, Piazza A, et al. Low-frequency transcranial magnetic stimulation in patients with cortical dysplasia. J Neurol 2003;250:761-762.

7. Brasil-Neto JP, De Araújo DP, Teixeira W, et al. Experimental therapy of epilepsy with transcranial magnetic stimulation: lack of additional benefit with prolonged treatment. Arq Neuropsiquiatr 2004;62:21-25.

8. Fregni F, Thome-Souza S, Bermpohl F, et al. Antiepileptic effects of repetitive transcranial magnetic stimulation in patients with cortical malformations: an EEG and clinical study. Stereotact Funct Neurosurg 2005;83:57-62.

9. Kinoshita M, Ikeda A, Begum T, et al. Low-frequency repetitive transcranial magnetic stimulation for seizure suppression in patients with extratemporal lobe epilepsy - a pilot study. Seizure 2005;14: 387-392.

10. Fregni F, Otachi PTM, Do Valle A, et al. A randomized clinical trial of repetitive transcranial magnetic stimulation in patients with refractory epilepsy. Ann Neurol 2006;60:447-455.

11. Cantello R, Rossi S, Varrasi C, et al. Slow repetitive TMS for drugresistant epilepsy: clinical and EEG findings of a placebo-controlled trial. Epilepsia 2007;48:366-374.

12. Joo EY, Han SJ, Chung SH, et al. Antiepileptic effects of lowfrequency repetitive transcranial magnetic stimulation by different stimulation durations and locations. Clin Neurophysiol 2007; 118:702-708.

13. Santiago-Rodríguez E, Cárdenas-Morales L, Harmony $\mathrm{T}$, et al. Repetitive transcranial magnetic stimulation decreases the number of seizures in patients with focal neocortical epilepsy. Seizure 2008; 17:677-683.

14. Sun W, Mao W, Meng X, et al. Low-frequency repetitive transcranial magnetic stimulation for the treatment of refractory partial epilepsy: a controlled clinical study. Epilepsia 2012;53:1782-1789.

15. Hsu W-Y, Cheng C-H, Lin M-W, et al. Antiepileptic effects of low frequency repetitive transcranial magnetic stimulation: a meta-analysis. Epilepsy Res 2011;96:231-240.

16. Kimiskidis VK, Kugiumtzis D, Papagiannopoulos S, et al. Transcranial magnetic stimulation (TMS) modulates epileptiform discharges in patients with frontal lobe epilepsy: a preliminary EEG-TMS study. Int J Neural Syst 2013;23:1250035:1-16.

17. Maizey L, Allen CPG, Dervinis M, et al. Comparative incidence rates of mild adverse effects to transcranial magnetic stimulation. Clin $\mathrm{Neu}$ rophysiol 2013;124:536-544.

18. Cramer JA, Perrine K, Devinsky O, et al. Development and cross-cultural translations of a 31-item quality of life in epilepsy inventory. Epilepsia 1998;39:81-88

19. Wassermann E, Epstein C, Ziemann U. Oxford handbook of transcranial stimulation. Oxford: Oxford University Press; 2008.

20. Fiest KM, Sajobi TT, Wiebe S. Epilepsy surgery and meaningful improvements in quality of life: results from a randomized controlled trial. Epilepsia 2014;55:886-892.

21. Ziemann U. TMS and drugs. Clin Neurophysiol 2004;115: 1717-1729.

22. Fregni F, Boggio PS, Valle AC, et al. Homeostatic effects of plasma valproate levels on corticospinal excitability changes induced by $1 \mathrm{~Hz}$ rTMS in patients with juvenile myoclonic epilepsy. Clin Neurophysiol 2006;117:1217-1227.

23. Ridding MC, Ziemann U. Determinants of the induction of cortical plasticity by non-invasive brain stimulation in healthy subjects. $J$ Physiol 2010;588:2291-2304.

24. Silvanto J. State-dependency of transcranial magnetic stimulation. Brain Topogr 2008;21:1-10.

25. Weisz N, Steidle L, Lorenz I. Formerly known as inhibitory: effects of 1-Hz rTMS on auditory cortex are state-dependent. Eur J Neurosci 2012;36:2077-2087. 


\section{Seynaeve et al.}

26. Rothkegel H, Sommer M, Paulus W. Breaks during $5 \mathrm{~Hz}$ rTMS are essential for facilitatory after effects. Clin Neurophysiol 2010;121:426-430.

27. Hamada M, Murase N, Hasan A, et al. The role of interneuron networks in driving human motor cortical plasticity. Cereb Cortex 2013;23:1593-1605.

28. Yi X, Fisher KM, Lai M, et al. Differences between Han Chinese and Caucasians in transcranial magnetic stimulation parameters. Exp Brain Res 2014;232:545-553.
29. Maeda F, Keenan JP, Tormos JM, et al. Interindividual variability of the modulatory effects of repetitive transcranial magnetic stimulation on cortical excitability. Exp Brain Res 2000;133:425430.

30. Rossi S, Hallett M, Rossini PM, et al. Safety, ethical considerations, and application guidelines for the use of transcranial magnetic stimulation in clinical practice and research. Clin Neurophysiol 2012;120:323-330. 